\title{
Renal Tubular Transport of Proline, Hydroxyproline, and Glycine in Health and in Familial Hyperprolinemia *
}

\author{
Charles R. Scriver, $\uparrow$ Mary L. Efron, and Irwin A. Schafer \\ (From the Department of Pediatrics, McGill University, and the Montreal Children's Hos- \\ pital, Montreal, Canada; the Department of Pediatrics, Harvard University \\ Medical School, Boston, Mass.; and the Department of Pediatrics, \\ Stanford University School of Medicine, Palo Alto, Calif.)
}

In man (2) and other mammals (3), most of the amino acid load in the glomerular filtrate is reabsorbed from the proximal tubule against a chemical gradient $(4,5)$. There are indications that human tubular absorption of the different chemical groups of amino acids occurs by means of several transport mechanisms. In cystinuria, for example, transport of only the di-amino acids, cystine, lysine, ornithine, and arginine, is impaired (6-9). In familial glycinuria (10) only glycine reabsorption is abnormal. In Hartnup disease $(11,12)$ there is impaired transport of most of the neutral amino acids ; there is, however, relatively little impairment in absorption of the cystinuric group of amino acids or of the imino acids and glycine.

The presence, in patients with familial hyperprolinemia $(13,14)$, of a specific hyperaminoaciduria comprising proline, hydroxyproline, and glycine raised the possibility of a transport mechanism selective for these three compounds. In this disease we observed that prolinuria occurred only when the plasma proline concentration was greater than $0.8 \mathrm{mM}$ (normal, $<0.3 \mathrm{mM}$ ); at this point hydroxyprolinuria and increased glycine excretion also appeared despite normal plasma concentrations of the latter two compounds. Presumably under these circumstances, the amount of proline in the glomerular filtrate exceeded its ca-

* Submitted for publication May 16, 1963 ; accepted November 5, 1963.

Presented in part to the American Society for Clinical Investigation, Atlantic City, N. J., April 1961(1). This investigation was supported by research grants A-5117, H-4706, RG-6742, and GM-09179-02 from the National Institutes of Health, Bethesda, Md.; by grant MA-1085 from the Medical Research Council, Ottawa, Canada; and by a grant from the United Cerebral Palsy Research and Education Foundation.

† Markle Scholar in Medical Science. pacity for complete tubular absorption. To account for the presence of hydroxyprolinuria and excessive glycinuria in the presence of prolinuria, the suggestion was made that proline competed with the other imino acid and with glycine for a "common"transport system in the renal tubule $(1,13)$.

The present study was undertaken to further clarify the relationships between imino acid and glycine transport. We also attempted to determine whether there was a maximal rate for tubular reabsorption of L-proline (TmPro). Our earlier assumption $(1,13)$ that imino acids and glycine shared a "common"transport mechanism was re-evaluated in light of the more recent investigations.

\section{Methods}

Subjects included seven healthy adult males, ages 24 to 40 years; one female child (S.McE.), age 12 years, with familial hyperprolinemia; her mother, age 39 years; her father, age 40 years; and one male adolescent (P.St.L.), age 16 years, with hypophosphatemic-glycinuric-glucosuric rickets.

Techniques. All experiments were performed in the morning, on fasted, resting subjects. Before and during the procedures, water was drunk to insure urine flow rates of 5 to $20 \mathrm{ml}$ per minute. Urine was collected by spontaneous voiding.

A) Rapid iv infusion experiments. A 30-minute (control) urine specimen was collected before infusion; at the mid-point of this period a venous blood sample was collected in heparin. The amino acid solution (0.65 mmoles per $\mathrm{kg}$ ) was then infused into an antecubital vein within 3 minutes. Urine specimens were collected during three consecutive clearance periods of 15 , 30 , and 30 minutes, respectively. At the mid-point of each clearance period $(8,30$, and 60 minutes $)$, venous blood was drawn. Endogenous creatinine clearance rates were determined in each period of two experiments. Eight infusions with L-proline, two with hydroxy-L-proline, three with glycine, two with L-alanine, and one with L-valine were performed. 
B) Steady-state iv infusion experiments. L-Proline was infused intravenously, using a constant-rate infusion pump, five times in three normal male subjects and once in the subject with hypophosphatemic rickets. Priming and sustaining doses of inulin were administered in the usual manner. After equilibration for 45 minutes, two consecutive urine collections, each lasting 15 to 20 minutes, were performed, and venous blood samples were drawn at two or three points during the double period. The true mid-point blood time was calculated with reference to each urine collection, assuming a delay between formation of urine at the glomerulus and collection from the bladder of less than 5 minutes at urine flow rates greater than $5 \mathrm{ml}$ per minute. Priming doses of L-proline ( 0.5 to $1.3 \mathrm{mmoles}$ per $\mathrm{kg}$ ) were then infused into the venous catheter delivering inulin, and a sustaining infusion of proline ( $3.2 \mu$ moles per $\mathrm{kg}$ per minute) was added to the sustaining inulin infusion. At least 20minute equilibration followed each proline priming infusion, after which a pair of 15-minute urine collections or a single longer collection was made with two accompanying venous blood samples. The priming infusions were repeated at intervals until the plasma proline concentration had been raised sufficiently to induce marked prolinuria.

Preparation of samples. Urine specimens were preserved with thymol and frozen at $-21^{\circ} \mathrm{C}$. Plasma was separated immediately from the blood cells, deproteinized with $1 \%$ picric acid (15), and frozen.

Materials. The amount of chromatographically pure L-amino acid ${ }^{1}$ required for each infusion was dissolved in 25 to $50 \mathrm{ml}$ distilled water, adjusted to $\mathrm{pH} 7.4$, passed through a Seitz (VF) filter, and steam autoclaved at 15 pounds pressure for 20 minutes at $122^{\circ} \mathrm{C}$. A sample was chromatographed before infusion to confirm purity.

Analytical methods. Urine samples, equivalent to 4-second volumes, and deproteinized plasma (625 $\mu \mathrm{l})$ were analyzed qualitatively for amino acid content. The method used was two-dimensional partition chromatography on filter paper, with phenol-ammonia and lutidine development (16), or high voltage electrophoresis on filter paper, followed by solvent partition chromatography in the second dimension (17). The chromatograms were stained with either Ninhydrin $(0.2 \% \mathrm{wt} / \mathrm{vol})$ in acetone, the Ninhydrin-isatin stain of Kolor and Roberts (18), or K. N. F. Shaw's modification of the latter $(0.25 \%$ wt/vol Ninhydrin, $0.020 \% \mathrm{wt} / \mathrm{vol}$ isatin; $1.0 \% \mathrm{vol} / \mathrm{vol}$ 2,6-lutidine in acetone, heated once at $75^{\circ} \mathrm{C}$ and 24 hours later at $100^{\circ} \mathrm{C}$ ).

1 Obtained from Mann Research, New York, N. Y.; California Biochemical Corp., Los Angeles, Calif.; Nutritional Biochemicals, Cleveland, Ohio; and Light and Co., Colnbrook, England.

TABLE I

Response to rapid infusion of imino acids

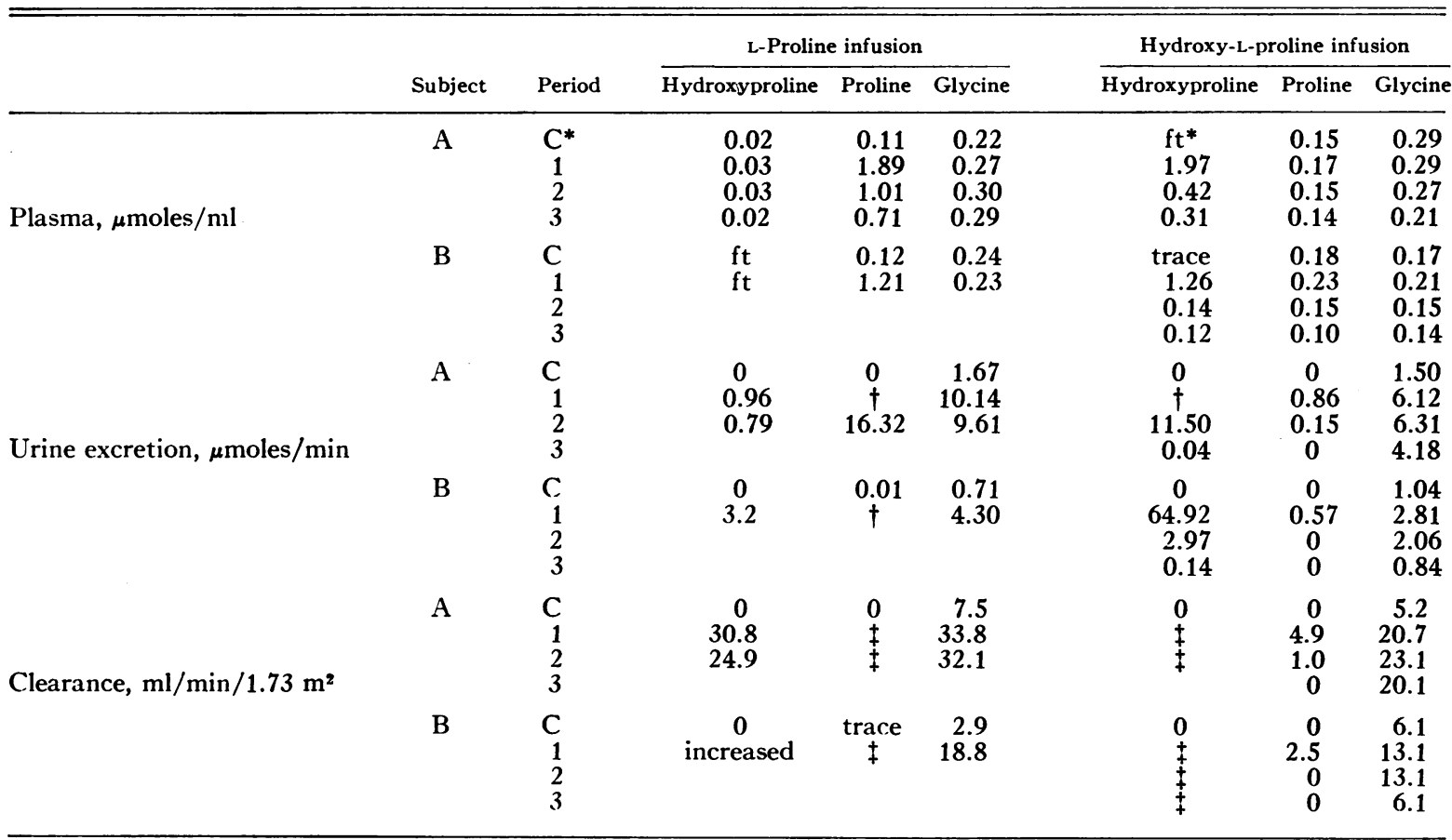

$* \mathrm{C}=$ control, $\mathrm{ft}=$ faint trace.

† Immeasurably high value.

‡ Value not calculated, since plasma and urine concentrations were changing rapidly. 
One-minute volumes of urine and suitable volumes of plasma were analyzed quantitatively by ion exchange column chromatography, using the earlier method of Moore and Stein (15) with their later modification $(19,20)$.

Free proline in urine and plasma samples from the constant-rate infusion experiments was also measured by the chemical method of Messer (21). This method is reported to obtain good specificity for proline with the color reaction. We encountered difficulties with urine samples' containing large amounts of proline, glycine, and hydroxyproline; therefore we performed ion exchange column chromatography $(19,20)$ on all samples at high levels of infusion. Proline values by the chemical method changed in a consistent manner after steadystate infusions but had poor predictable numerical relation to values obtained by chromatography $(19,20)$.

Inulin was measured by the modification of Schreiner's technique (22) and endogenous creatinine by the method of Miller (23).

\section{Results}

Rapid iv infusion experiments in normal subjects. $^{2}$ A) L-proline. After infusion, there was a marked increase in urinary excretion of proline, hydroxyproline, and glycine in all subjects. Other amino acids were not comparably affected. The changes were most obvious in the first postinfusion period ( 0 to 15 minutes) and subsided thereafter, as the plasma proline concentration fell (Table I). The increase in urinary excretion rates of hydroxyproline and glycine were not associated with increases in their plasma concentrations, nor with increases in the filtered load when monitored by endogenous creatinine clearance rates. Therefore, clearance rates of hydroxyproline and glycine could be raised by increasing the filtered load of proline (Figure 1).

There were slight increases noted in the urinary excretion of threonine, serine, methionine, tyrosine, and phenylalanine in three subjects; this was observed only during the second or third postinfusion period. Amino acid clearance rates were determined in two subjects; the values remained within normal limits, in contrast to the changes in renal clearance of hydroxyproline and glycine. The endogenous creatinine clearance rate did not change significantly in one subject in whom it was measured before and after infusion.

2 The complete quantitative data for all experiments analyzed by column chromatography are not included in the text but have been deposited with the American Documentation Institute of the Library of Congress.
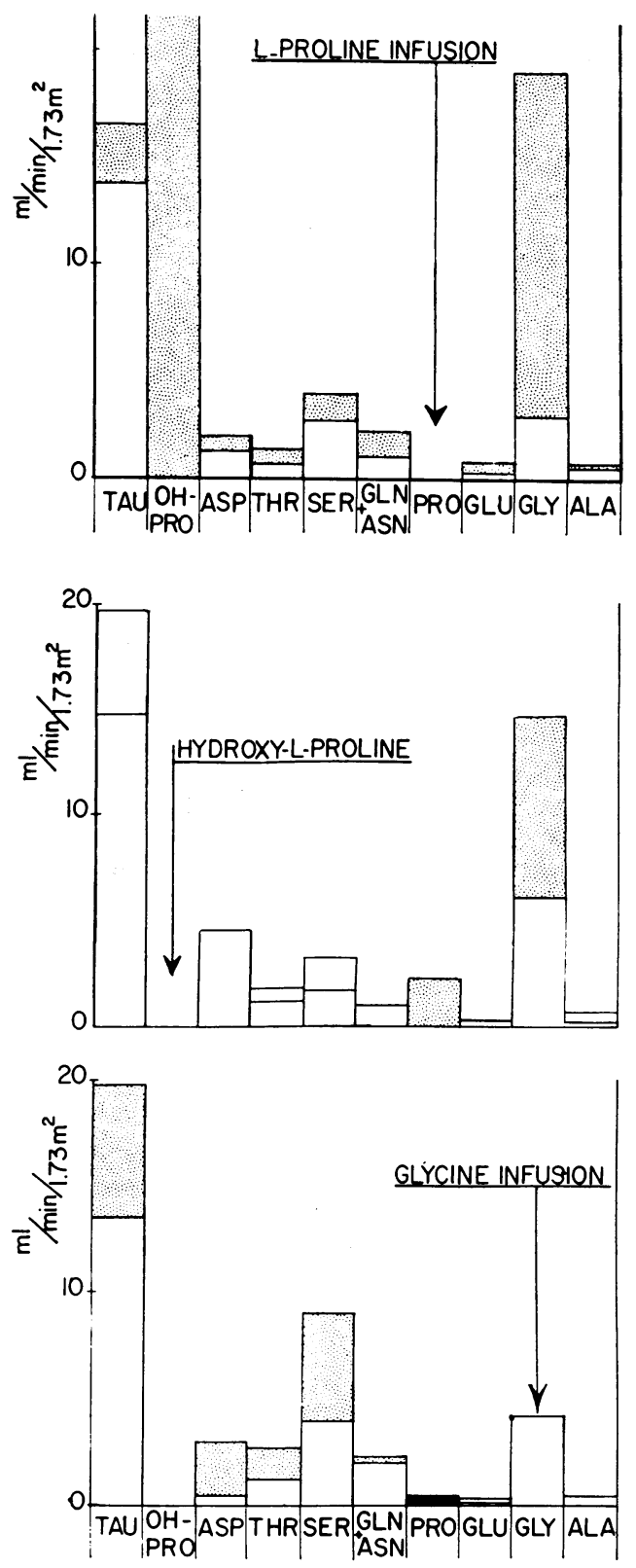

Fig. 1. Endogenous amino ACID RENAL Clearance RATES IN A SINGLE SUBJECT, BEFORE AND AFTER (0 TO 15 MINUTES) RAPID INFUSION OF AN AMINO ACID. Upper figure: response to L-proline infusion; middle figure: response to hydroxy-L-proline infusion; lower figure: response to glycine infusion. Stippling represents an increase in clearance rates after infusion; otherwise the lower horizontal line in the histogram represents a reduction in clearance value after infusion. TAU $=$ taurine, $\mathrm{OH}-\mathrm{PRO}=$ hydroxyproline, $\mathrm{ASP}=$ aspartic acid, THR $=$ threonine, $\mathrm{SER}=$ serine, $\mathrm{GLN}=$ glutamine, $\mathrm{ASN}=$ asparagine, $\mathrm{PRO}=$ proline, $\mathrm{GLU}=$ glutamic acid, GLY $=$ glycine, $\mathrm{ALA}=$ alanine. 
B) Hydroxy-L-proline. Urinary excretion rates and endogenous renal clearance rates of proline and glycine were increased by iv infusion of hydroxyproline (Table I, Figure 1). The response was greatest when plasma hydroxyproline concentration was highest. The effect on proline and glycine clearance was less than the comparable effect at equivalent plasma levels for proline after its infusion.

C) Glycine. Three infusions were performed, two on the same subject. There was no hydroxyprolinuria produced on any occasion. On a single occasion there was slight but clearly detectable prolinuria in the first postinfusion period (Figure 1); preinfusion excretion and clearance of proline rates were zero, respectively; postinfusion, $0.050 \mu$ mole per minute and $0.3 \mathrm{ml}$ per minute per $1.73 \mathrm{~m}^{2}$, respectively. Postinfusion prolinuria was not clearly demonstrable in the other two studies.

In both subjects, glycine infusion was followed by increased urinary excretion of threonine and serine (Figure 1). The renal clearance rates of these compounds increased; their raised excretion rates were not primarily accounted for by changes in plasma concentration. We also observed a twofold increase in $\alpha$-amino-n-butyric and histidine clearances and marked increase in sarcosine concentration of plasma in one experiment.

D) L-valine and L-alanine. Infusion of either amino acid, producing plasma concentrations of the infused compound equivalent to those obtained in the other studies, had no effect on imino acid excretion. Valine infusion increased the glycine renal clearance from $6.6 \mathrm{ml}$ per minute per 1.73 $\mathrm{m}^{2}$ (normal) to $12.1 \mathrm{ml}$ per minute per $1.73 \mathrm{~m}^{2}$

TABLE II

$C_{A A}$ with rapid infusion of amino acids in normal adults*

\begin{tabular}{|c|c|c|c|c|c|c|c|c|c|}
\hline \multirow[b]{3}{*}{ Amino acid } & \multicolumn{4}{|c|}{ Control values (preinfusion) $\dagger$} & \multicolumn{5}{|c|}{ Postinfusion values $(0-15$-minutes $) \ddagger$} \\
\hline & \multicolumn{2}{|c|}{ Range } & \multirow{2}{*}{\multicolumn{2}{|c|}{ Grand mean $\pm S D$}} & \multirow{2}{*}{$\begin{array}{c}\text { L-Proline } \\
\text { infusion }\end{array}$} & \multirow{2}{*}{$\begin{array}{l}\text { Hydroxy- } \\
\text { L-proline } \\
\text { infusion }\end{array}$} & \multirow{2}{*}{$\begin{array}{c}\text { Glycine } \\
\text { infusion }\end{array}$} & \multirow{2}{*}{$\begin{array}{l}\text { L-Valine } \\
\text { infusion }\end{array}$} & \multirow{2}{*}{$\begin{array}{c}\text { L-Alanine } \\
\text { infusion }\end{array}$} \\
\hline & Group 1 & Group 2 & & & & & & & \\
\hline Proline & 0 & $0-0.05$ & & 0 & & $\begin{array}{l}4.94 \\
2.52\end{array}$ & $\begin{array}{l}0.12 \S \\
0.23\end{array}$ & $\begin{array}{l}0 \\
0\end{array}$ & $\begin{array}{l}0 \\
0\end{array}$ \\
\hline Hydroxyproline & 0 & 0 & & 0 & $\begin{array}{l}30.8 \\
37.6\end{array}$ & & $\begin{array}{l}0 \\
0\end{array}$ & $\begin{array}{l}0 \\
0\end{array}$ & $\begin{array}{l}0 \\
0\end{array}$ \\
\hline Glycine & $2.7-5.8$ & $2.5-6.6$ & 4.7 & \pm 1.3 & $\begin{array}{l}33.8 \\
18.8\end{array}$ & $\begin{array}{l}20.7 \\
13.3\end{array}$ & & 12.10 & 5.80 \\
\hline Valine & $0.04-0.30$ & $0.15-0.21$ & 0.16 & \pm 0.06 & 0.14 & $\begin{array}{l}0.13 \\
0.07\end{array}$ & $\begin{array}{l}0.04 \\
0.13\end{array}$ & & 0.13 \\
\hline Alanine & $0.3-0.9$ & $0.2-0.8$ & 0.50 & \pm 0.21 & $\begin{array}{l}0.3 \\
0.9\end{array}$ & $\begin{array}{l}0.39 \\
0.59\end{array}$ & & 0.44 & \\
\hline Glutamic acid & $0.3-0.7$ & $0.1-0.8$ & 0.4 & \pm 0.25 & 0.6 & $\begin{array}{l}0.17 \\
0.33\end{array}$ & $\begin{array}{l}0.55 \\
0.10\end{array}$ & trace & 0.80 \\
\hline Threonine & $0.8-1.5$ & $0.6-1.6$ & 1.0 & \pm 0.35 & $\begin{array}{l}0.45 \\
1.70\end{array}$ & $\begin{array}{l}1.61 \\
1.26\end{array}$ & $\begin{array}{l}1.62 \\
2.71\end{array}$ & 1.40 & 0.60 \\
\hline$\alpha$-Amino-n-butyric acid & $0-1.3$ & $0.3-1.5$ & 1.1 & \pm 0.46 & 0.52 & 1.12 & $\begin{array}{l}1.12 \\
\text { trace }\end{array}$ & 1.83 & 0.61 \\
\hline Leucine & $0.2-0.9$ & $0.2-0.3$ & 0.3 & \pm 0.23 & 0.34 & $\begin{array}{l}0.38 \\
0.25\end{array}$ & 0.26 & 0.23 & 0.38 \\
\hline Phenylalanine & $0.7-1.4$ & $0.3-1.1$ & 0.87 & \pm 0.3 & 1.32 & $\begin{array}{l}0.85 \\
1.22\end{array}$ & 0.62 & 0.90 & 1.00 \\
\hline Histidine & $4.7-9.7$ & $3.3-8.4$ & 6.5 & \pm 2.02 & & 3.3 & $\begin{array}{r}4.46 \\
17.90\end{array}$ & & 8.25 \\
\hline Lysine & $0.2-1.9$ & $0.2-0.7$ & 0.7 & \pm 0.6 & & 0.3 & $\begin{array}{l}0.8 .3 \\
1.6\end{array}$ & & 0.52 \\
\hline
\end{tabular}

* $\mathrm{C}_{\mathrm{AA}}=$ clearance of amino acids, expressed as $\mathrm{ml}$ per minute per $1.73 \mathrm{~m}^{2}$.

$\dagger$ Controls: Group 1, subjects studied by Cusworth and Dent (2) (no. = 4); Group 2, normal subjects (no. = 5) from present study.

$\ddagger$ Postinfusion data: When two values are given, they represent data from two different studies on different subjects.

$\$$ These studies were in the same subject. In the first study ( 0.12 value), the sample was analyzed on two different Beckman amino acid analyzers; no proline was found in one analysis. 
(abnormal value). Despite the relation in steric conformations, valine had no influence on leucine and isoleucine excretion; the excretion of $\beta$-aminoisobutyric acid, a potential product of $L$-valine metabolism (24), was not increased by valine infusion.

The excretion response to rapid infusion of each of the five amino acids is summarized in

TABLE III

Constant infusion of L-proline*

\begin{tabular}{|c|c|c|c|c|c|c|c|}
\hline Amino acid & Periodt & Plasma & Urine & $\mathrm{C}_{\mathbf{A A}}$ & Filtered & Reabsorption & $\begin{array}{l}\text { Amount } \\
\text { reabsorbed }\end{array}$ \\
\hline & & Amoles $/ \mathrm{ml}$ & $\begin{array}{c}\mu m o l e s / \min / \\
1.73 \mathrm{~m}^{2}\end{array}$ & $\begin{array}{l}\mathrm{ml} / \mathrm{min} / \\
1.73 \mathrm{~m}^{2}\end{array}$ & $\begin{array}{c}\text { Mmoles } / \mathrm{min} / \\
1.73 \mathrm{~m}^{2}\end{array}$ & $\begin{array}{l}\text { Mmoles } / \min / \\
1.73 \mathrm{~m}^{2}\end{array}$ & $\%$ \\
\hline Ornithine & $\begin{array}{l}\mathrm{C} \\
4_{+}^{+}\end{array}$ & $\begin{array}{l}0.066 \\
0.075\end{array}$ & $\begin{array}{l}0.029 \\
0.048\end{array}$ & $\begin{array}{l}0.44 \\
0.64\end{array}$ & $\begin{array}{r}7.05 \\
10.24\end{array}$ & $\begin{array}{r}7.02 \\
10.19\end{array}$ & $\begin{array}{l}99.5 \\
99.3\end{array}$ \\
\hline Lysine & $\begin{array}{l}\mathrm{C} \\
4\end{array}$ & $\begin{array}{l}0.127 \\
0.134\end{array}$ & $\begin{array}{l}0.083 \\
0.136\end{array}$ & $\begin{array}{l}0.65 \\
1.0\end{array}$ & $\begin{array}{l}13.72 \\
18.50\end{array}$ & $\begin{array}{l}13.64 \\
18.36\end{array}$ & $\begin{array}{l}99.5 \\
99.3\end{array}$ \\
\hline Histidine & $\begin{array}{l}\mathrm{C} \\
4\end{array}$ & $\begin{array}{l}0.057 \\
0.071\end{array}$ & $\begin{array}{l}0.475 \\
1.080\end{array}$ & $\begin{array}{r}8.34 \\
15.20\end{array}$ & $\begin{array}{l}6.13 \\
9.80\end{array}$ & $\begin{array}{l}5.65 \\
8.72\end{array}$ & $\begin{array}{l}92.3 \\
89.0\end{array}$ \\
\hline Arginine & $\begin{array}{l}\mathrm{C} \\
4\end{array}$ & $\begin{array}{l}0.045 \\
0.058\end{array}$ & $\begin{array}{l}\text { trace } \\
\text { trace }\end{array}$ & $\begin{array}{l}\text { trace } \\
\text { trace }\end{array}$ & $\begin{array}{l}4.83 \\
8.00\end{array}$ & $\begin{array}{l}\sim 4.83 \\
\sim 8.0\end{array}$ & $\begin{array}{l}\sim 100 \\
\sim 100\end{array}$ \\
\hline Hydroxyproline & $\begin{array}{l}\mathrm{C} \\
4\end{array}$ & $\begin{array}{c}\sim 0.05 \\
0.042\end{array}$ & $\begin{array}{c}0 \\
1.64\end{array}$ & $\begin{array}{c}0 \\
32.8\end{array}$ & $\begin{array}{r}\sim 5.00 \\
5.80\end{array}$ & $\begin{array}{r}\sim 5.00 \\
4.16\end{array}$ & $\begin{array}{l}100 \\
71.8\end{array}$ \\
\hline Aspartic acid & $\begin{array}{l}\mathrm{C} \\
4\end{array}$ & $\begin{array}{l}0.006 \\
0.010\end{array}$ & $\begin{array}{l}0.014 \\
0.017\end{array}$ & $\begin{array}{l}2.34 \\
1.70\end{array}$ & $\begin{array}{l}0.64 \\
1.38\end{array}$ & $\begin{array}{l}0.63 \\
1.36\end{array}$ & $\begin{array}{l}98.5 \\
98.5\end{array}$ \\
\hline Threonine & $\begin{array}{l}\mathrm{C} \\
4\end{array}$ & $\begin{array}{l}0.094 \\
0.112\end{array}$ & $\begin{array}{l}0.152 \\
0.448\end{array}$ & $\begin{array}{l}1.62 \\
4.00\end{array}$ & $\begin{array}{l}10.10 \\
15.46\end{array}$ & $\begin{array}{l}10.01 \\
15.01\end{array}$ & $\begin{array}{l}99.0 \\
97.3\end{array}$ \\
\hline Serine & $\begin{array}{l}\mathrm{C} \\
4\end{array}$ & $\begin{array}{l}0.099 \\
0.121\end{array}$ & $\begin{array}{l}0.323 \\
1.200\end{array}$ & $\begin{array}{l}3.27 \\
9.92\end{array}$ & $\begin{array}{l}10.62 \\
16.70\end{array}$ & $\begin{array}{l}10.30 \\
15.50\end{array}$ & $\begin{array}{l}97.0 \\
92.9\end{array}$ \\
\hline Glutamine and asparagine & $\begin{array}{l}\mathrm{C} \\
4\end{array}$ & $\begin{array}{l}0.478 \\
0.302\end{array}$ & $\begin{array}{l}0.429 \\
1.506\end{array}$ & $\begin{array}{l}0.90 \\
1.70\end{array}$ & $\begin{array}{l}51.30 \\
41.70\end{array}$ & $\begin{array}{l}50.82 \\
40.20\end{array}$ & $\begin{array}{l}99.0 \\
96.5\end{array}$ \\
\hline Proline & $\begin{array}{l}\mathrm{C} \\
4\end{array}$ & $\begin{array}{l}0.152 \\
1.565\end{array}$ & $\begin{array}{l}\text { trace } \\
56.6\end{array}$ & $\begin{array}{l}\text { trace } \\
36.95\end{array}$ & $\begin{array}{l}16.31 \\
216.0\end{array}$ & $\begin{array}{c}\sim 16.31 \\
159.4\end{array}$ & $\begin{array}{r}\sim 100 \\
73.9\end{array}$ \\
\hline Glutamic acid & $\begin{array}{l}C \\
4\end{array}$ & $\begin{array}{l}0.084 \\
0.211\end{array}$ & $\underset{\S}{0.034}$ & 0.40 & $\begin{array}{r}9.02 \\
29.10\end{array}$ & 8.99 & 99.8 \\
\hline Glycine & $\begin{array}{l}\mathrm{C} \\
4\end{array}$ & $\begin{array}{l}0.216 \\
0.202\end{array}$ & $\begin{array}{r}1.00 \\
10.68\end{array}$ & $\begin{array}{r}4.63 \\
52.80\end{array}$ & $\begin{array}{l}23.20 \\
27.85\end{array}$ & $\begin{array}{l}22.20 \\
17.17\end{array}$ & $\begin{array}{l}96.7 \\
61.5\end{array}$ \\
\hline Alanine & $\begin{array}{l}\mathrm{C} \\
4\end{array}$ & $\begin{array}{l}0.290 \\
0.360\end{array}$ & $\begin{array}{l}0.165 \\
0.804\end{array}$ & $\begin{array}{l}0.57 \\
2.24\end{array}$ & $\begin{array}{l}31.08 \\
49.70\end{array}$ & $\begin{array}{l}30.92 \\
48.90\end{array}$ & $\begin{array}{l}99.2 \\
98.4\end{array}$ \\
\hline$\alpha$-Amino-n-butyric acid & $\begin{array}{l}\mathrm{C} \\
4\end{array}$ & $\begin{array}{l}0.017 \\
0.017\end{array}$ & $\begin{array}{l}0.030 \\
0.090\end{array}$ & $\begin{array}{l}1.77 \\
5.30\end{array}$ & $\begin{array}{l}1.83 \\
2.34\end{array}$ & $\begin{array}{l}1.80 \\
2.25\end{array}$ & $\begin{array}{l}98.4 \\
96.4\end{array}$ \\
\hline Valine & $\begin{array}{l}\mathrm{C} \\
4\end{array}$ & $\begin{array}{l}0.184 \\
0.145\end{array}$ & $\begin{array}{l}0.016 \\
0.076\end{array}$ & $\begin{array}{l}0.09 \\
0.50\end{array}$ & $\begin{array}{l}19.78 \\
20.00\end{array}$ & $\begin{array}{l}19.76 \\
19.92\end{array}$ & $\begin{array}{l}99.9 \\
99.5\end{array}$ \\
\hline Half-cystine & $\begin{array}{l}\mathrm{C} \\
4\end{array}$ & & $\begin{array}{l}0.029 \\
0.112\end{array}$ & & & & \\
\hline Methionine & $\begin{array}{l}C \\
4\end{array}$ & $\begin{array}{l}0.019 \\
0.015\end{array}$ & $\begin{array}{l}0.012 \\
0.038\end{array}$ & $\begin{array}{l}0.63 \\
2.52\end{array}$ & $\begin{array}{l}2.04 \\
2.07\end{array}$ & $\begin{array}{l}2.03 \\
2.03\end{array}$ & $\begin{array}{l}99.5 \\
98.0\end{array}$ \\
\hline Isoleucine & $\begin{array}{l}\mathrm{C} \\
4\end{array}$ & $\begin{array}{l}0.042 \\
0.041\end{array}$ & $\begin{array}{l}0.021 \\
0.052\end{array}$ & $\begin{array}{l}0.50 \\
1.26\end{array}$ & $\begin{array}{l}4.51 \\
5.66\end{array}$ & $\begin{array}{l}4.49 \\
5.61\end{array}$ & $\begin{array}{l}99.4 \\
99.2\end{array}$ \\
\hline Leucine & $\begin{array}{l}\mathrm{C} \\
4\end{array}$ & $\begin{array}{l}0.093 \\
0.090\end{array}$ & $\begin{array}{l}0.030 \\
0.052\end{array}$ & $\begin{array}{l}0.32 \\
0.58\end{array}$ & $\begin{array}{l}10.00 \\
12.42\end{array}$ & $\begin{array}{r}9.97 \\
12.37\end{array}$ & $\begin{array}{l}99.6 \\
99.4\end{array}$ \\
\hline Tyrosine & $\begin{array}{l}C \\
4\end{array}$ & $\begin{array}{l}0.045 \\
0.042\end{array}$ & $\begin{array}{l}0.077 \\
0.148\end{array}$ & $\begin{array}{l}1.72 \\
3.52\end{array}$ & $\begin{array}{l}4.84 \\
5.80\end{array}$ & $\begin{array}{l}4.77 \\
5.65\end{array}$ & $\begin{array}{l}98.4 \\
97.5\end{array}$ \\
\hline Phenylalanine & $\begin{array}{l}\mathrm{C} \\
4\end{array}$ & $\begin{array}{l}0.044 \\
0.042\end{array}$ & $\begin{array}{l}0.049 \\
0.103\end{array}$ & $\begin{array}{l}1.11 \\
2.48\end{array}$ & $\begin{array}{l}4.72 \\
5.80\end{array}$ & $\begin{array}{l}4.67 \\
5.70\end{array}$ & $\begin{array}{l}98.8 \\
97.5\end{array}$ \\
\hline
\end{tabular}

* Subject, normal adult male.

† Inulin clearance in control period (C), $107.3 \mathrm{ml}$ per minute per $1.73 \mathrm{~m}^{2}$; in period $4,138.0 \mathrm{ml}$ per minute per $1.73 \mathrm{~m}^{2}$. $\$$ At TmPro; 20-minute period beginning 135 minutes after onset of L-proline infusion.

$\S$ Overrun by proline. 
TABLE IV

Tubular absorption of $L$-proline and excretion rates of glycine and hydroxyproline in relation to filtered $L$-proline load

\begin{tabular}{|c|c|c|c|c|c|c|c|c|}
\hline Subject & Time & $\begin{array}{l}\text { Inulin } \\
\text { clearance }\end{array}$ & $\begin{array}{l}\text { Plasma } \\
\text { proline }\end{array}$ & $\begin{array}{c}\text { Filtered } \\
\text { proline }\end{array}$ & $\begin{array}{l}\text { Urinary } \\
\text { proline }\end{array}$ & $\begin{array}{l}\text { Tubular } \\
\text { absorption } \\
\text { of proline }\end{array}$ & $\begin{array}{l}\text { Urinary } \\
\text { glycine }\end{array}$ & $\begin{array}{r}\text { Urinary } \\
\text { hydroxy- } \\
\text { proline }\end{array}$ \\
\hline & & $m l / m i n$ & Mmoles $/ m l$ & $\underset{\min }{\mu \text { moles/ }}$ & Mmoles/min & $\underset{\min }{\mu \text { moles } /}$ & $\mu$ moles $/ \min$ & $\underset{\min }{\mu \text { moles } /}$ \\
\hline \multicolumn{9}{|c|}{ Part I (priming dose of proline, $50 \mathrm{mg} / \mathrm{kg}$ ) } \\
\hline $\begin{array}{l}24 \text { years } \\
65 \mathrm{~kg}\end{array}$ & $\begin{array}{c}30-61 \\
85-105 \\
130-159 \\
175-206\end{array}$ & $\begin{array}{l}118 \\
106 \\
125 \\
114\end{array}$ & $\begin{array}{l}0.12 \\
0.50 \\
0.96 \\
1.15\end{array}$ & $\begin{array}{r}14 \\
53 \\
120 \\
132\end{array}$ & $\begin{array}{r}0^{*} \\
0^{*} \\
<1.0 \\
1.0\end{array}$ & $\begin{array}{r}14 \\
53 \\
119 \\
138\end{array}$ & $\begin{array}{c}\dagger \\
\mathrm{nc}_{+}^{+} \\
\mathrm{nc}_{+}^{+} \\
1.27\end{array}$ & $\begin{array}{l}0 \pm \\
0 \pm \\
0 \ddagger \\
0\end{array}$ \\
\hline \multicolumn{9}{|c|}{ Part II (priming dose of proline, $150 \mathrm{mg} / \mathrm{kg}$ ) } \\
\hline & $\begin{array}{c}40-89 \\
111-140 \\
159-192\end{array}$ & $\begin{array}{r}117 \\
108 \\
81\end{array}$ & $\begin{array}{l}2.2 \\
3.9 \\
3.5\end{array}$ & $\begin{array}{l}260 \\
420 \\
285\end{array}$ & $\begin{array}{r}73 \\
260 \\
86\end{array}$ & $\begin{array}{l}187 \\
160 \\
199\end{array}$ & $\begin{array}{l}6.2 \\
9.9 \\
6.9\end{array}$ & $\begin{array}{l}0.8 \\
1.6 \\
1.0\end{array}$ \\
\hline \multicolumn{9}{|c|}{ (Priming dose of proline, $50 \mathrm{mg} / \mathrm{kg}$ ) } \\
\hline $\begin{array}{l}31 \text { years } \\
71 \mathrm{~kg}\end{array}$ & $\begin{array}{c}30-60 \\
75-91 \\
108-125 \\
141-156 \\
175-190\end{array}$ & $\begin{array}{r}105 \\
97 \\
108 \\
140 \\
138\end{array}$ & $\begin{array}{l}0.15 \\
0.65 \\
0.88 \\
1.27 \\
1.56\end{array}$ & $\begin{array}{r}16 \\
64 \\
95 \\
178 \\
216\end{array}$ & $\begin{array}{l}<.005 \\
0^{*} \\
0.7 \\
14 \\
57\end{array}$ & $\begin{array}{r}16 \\
64 \\
94 \\
164 \\
159\end{array}$ & $\begin{array}{r}1.0 \\
\mathrm{nc}+ \\
2.2 \\
10.7\end{array}$ & $\begin{array}{l}0 \\
0 \ddagger \\
0 \\
0.44\end{array}$ \\
\hline \multicolumn{9}{|c|}{ Study $A$ (priming dose of proline, $100 \mathrm{mg} / \mathrm{kg}$ ) } \\
\hline $\begin{array}{l}26 \text { years } \\
50 \mathrm{~kg}\end{array}$ & $\begin{array}{c}\text { Preinfusion } \\
50-75 \\
110-127 \\
157-173\end{array}$ & $\begin{array}{l}115 \\
125 \\
113\end{array}$ & $\begin{array}{l}0.16 \\
0.97 \\
1.12 \\
2.14\end{array}$ & $\begin{array}{l}112 \\
140 \\
242\end{array}$ & $\begin{array}{l}0^{*} \\
0.5 \\
14 \\
55\end{array}$ & $\begin{array}{l}112 \\
126 \\
177\end{array}$ & $\begin{array}{c}\dagger \\
1.6 \\
6.3\end{array}$ & $\begin{array}{c}0 \ddagger \\
0 \\
0.38 \\
0.66\end{array}$ \\
\hline \multicolumn{9}{|c|}{ Study $B$ (priming dose of proline, $150 \mathrm{mg} / \mathrm{kg}$ ) } \\
\hline & $\begin{array}{c}\text { Preinfusion } \\
45-85 \\
115-136 \\
170-190 \\
220-240\end{array}$ & $\begin{array}{l}125 \\
129 \\
140 \\
128\end{array}$ & $\begin{array}{l}0.72 \\
2.03 \\
2.44 \\
2.80\end{array}$ & $\begin{array}{r}90 \\
262 \\
342 \\
358\end{array}$ & $\begin{array}{r}0 \ddagger \\
0 \ddagger \\
15 \\
72 \\
104\end{array}$ & $\begin{array}{r}90 \\
247 \\
270 \\
254\end{array}$ & $\begin{array}{c}\dagger \\
\text { nc } \\
4.4 \\
6.4\end{array}$ & $\begin{array}{l}0 \ddagger \\
0 \ddagger \\
0.34 \\
0.62 \\
0.71\end{array}$ \\
\hline $\begin{array}{l}\text { 4: } \text { Male } \\
16 \text { years } \\
\text { Hypophosphatemic rickets } \\
\text { with hyperglycinuria }\end{array}$ & $\begin{array}{c}45-78 \\
116-133 \\
150-171\end{array}$ & $\begin{array}{l}72 \\
76 \\
72\end{array}$ & $\begin{array}{l}0.15 \\
0.93 \\
1.38\end{array}$ & $\begin{array}{l}11 \\
71 \\
99\end{array}$ & $\begin{array}{l}0.09 \\
0.23 \\
9.7\end{array}$ & $\begin{array}{l}11 \\
70 \\
89\end{array}$ & $\begin{array}{l}3.3 \\
4.3 \\
7.2\end{array}$ & $\begin{array}{l}\text { trace } \\
0.10 \\
0.75\end{array}$ \\
\hline
\end{tabular}

* Subject 1: Two studies, Tm not reached in Part I; plotted as one study in Figure 2. Subject 3: Two studies apparent and true Tm reached in both. Values differed. Plotted as two studies in Figure 2.

+ Normal amount of glycine excreted (qualitative estimate).

$\ddagger$ Observation made by qualitative chromatographic methods; $n c=$ no change from previous period (qualitative method).

Table II. Endogenous renal clearance data are presented for the amino acids used for infusion and also for one amino acid representing each of the major chemical groups [these include neutral amino acids (C2 to C5); a hydroxy-C4-homologue (threonine); and an aromatic, a heterocyclic, a cationic, and an anionic amino acid]. Data for the first postinfusion period are compared with control data; the latter include preinfusion amino acid clearance values from the present study and from another study using comparable techniques in normal adults (2). Also evident in Table II are the selective interrelations between imino acid and glycine during renal clearance.

Constant-rate infusion studies with $L$-proline. Four subjects were studied six times to determine whether the increases in urinary excretion and renal clearance rates of hydroxyproline and glycine, following L-proline infusion, were the result of changes in the filtered load, of net tubular secretion, or of inhibition of tubular absorption of these compounds.

The increase in urinary excretion of hydroxyproline and glycine was clearly related to selective inhibition of tubular absorption of these com- 
TABLE V

Maximal tubular absorption of L-proline in man

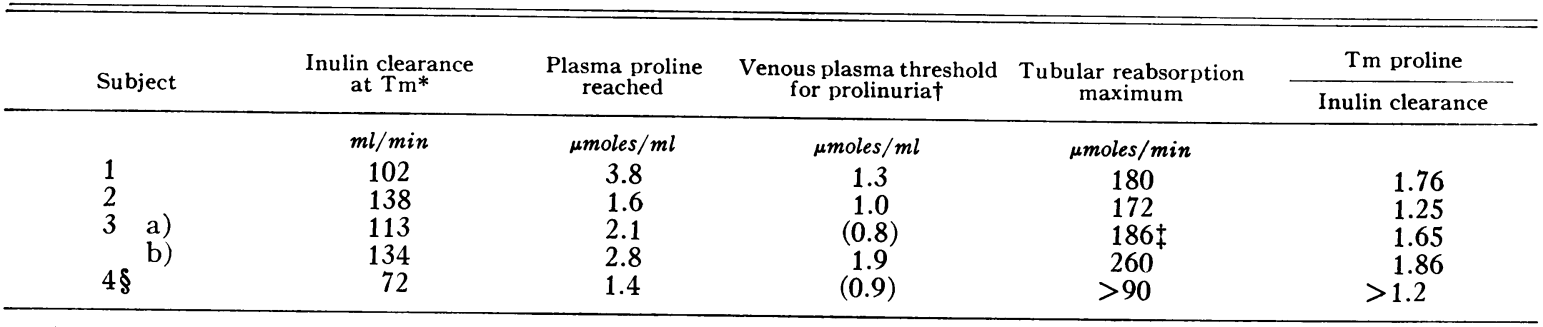

* Average at Tm. values.

† "Line threshold" method; figures in parentheses indicate "line" method applied to data below Tm, hence lower

$\ddagger$ "Apparent" Tm value, based on the maximal reabsorbed load reached, which appeared near the Tm value.

$\$$ Tm not reached; data are values at reabsorptive capacity reached in experiment.

pounds; an example is shown in Table III. This phenomenon appeared in each subject when the filtered load of proline reached the threshold for prolinuria itself (Table IV); the inhibitory effect of proline increased as its filtered concentration increased. In addition to the obvious effect on hydroxyproline and glycine, there was slight inhibition of tubular absorption of threonine, serine, histidine, and glutamine plus asparagine.

Evidence for a Tm-proline in man. The four subjects discussed in the previous section were investigated for the presence of a maximal tubular reabsorptive capacity for L-proline (TmPro). Insignificant amounts of proline were excreted when the plasma proline concentration was normal ( 0.10 to $0.30 \mu$ mole per $\mathrm{ml}$ ) (Tables III and IV). Prolinuria did not increase significantly until the plasma proline concentration reached at least $0.8 \mu$ mole per $\mathrm{ml}$. The "line-threshold" 3 plasma concentration at which prolinuria occurred was approximately $1.0 \mu$ mole per $\mathrm{ml}$ in these studies (Table V).

A maximal rate for tubular reabsorption of L-proline was clearly observed in three studies. In a fourth, the filtered proline load was insufficient to obtain more than one point at the apparent $\mathrm{Tm}$. Since it was reasonable to believe that a Tm had actually been reached, the apparent TmPro is included with the data in Table V. These data are expressed in Figure 2 as the relation between the filtered load: Tm ratio and the reabsorbed load: Tm ratio; the data conform to the expected pattern for a $\mathrm{Tm}$ phenomenon.

\footnotetext{
${ }^{3}$ Calculated from the abscissa intercept of the regression line relating urinary excretion of proline (ordinate) to plasma proline concentration (abscissa) (25).
}

A TmPro was not reached in the rackitic subject at a reabsorbed load: inulin clearance ratio of $1: 2$. The "line-threshold" value for prolinuria in this subject was comparable to the values for the other subjects.

Imino acid and glycine excretion in familial hyperprolinemia. The apparent threshold for prolinuria in three sibs with familial hyperprolinemia and normal renal function was approximately 0.8 to $1.0 \mu$ mole per $\mathrm{ml}(13,14)$. The value was slightly lower in the proband who had severely

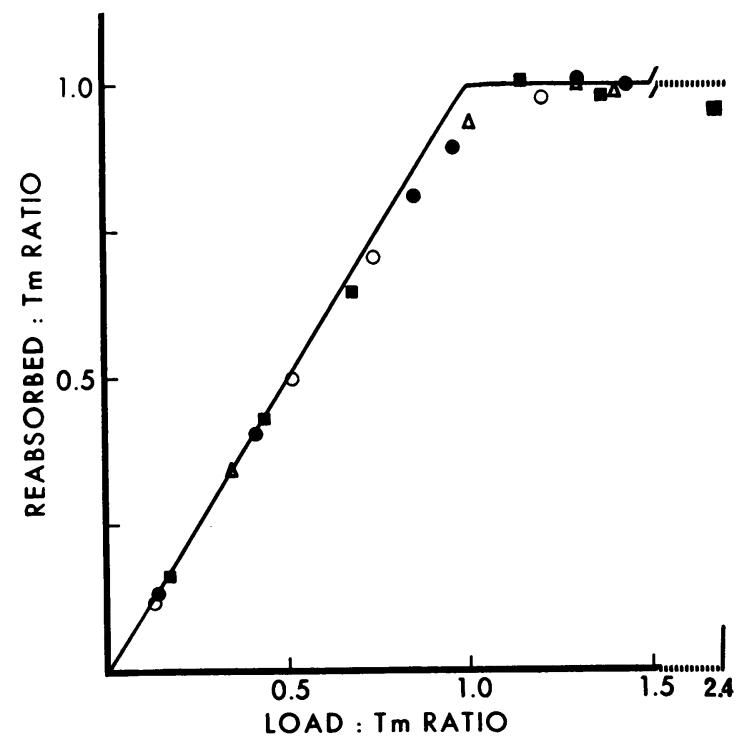

Fig. 2. RELATION OF FILTERED AND REABSORBED AMOUNTS OF L-PROLINE TO THE MAXIMAL RATE OF TUBULAR REABSORPTION (TR) OF PROLINE (TMPro) IN MAN. The maximal filtered load: TmPro ratio obtained was 2.38 , at which the TR: TmPro ratio was still approximately 1.0. Note splay in nephron response. Symbols refer to subjects in Table IV : - Subject 1 ; Subject 2; $0, \triangle$ Subject 3 , studies $\mathrm{A}$ and $\mathrm{B}$, respectively. 


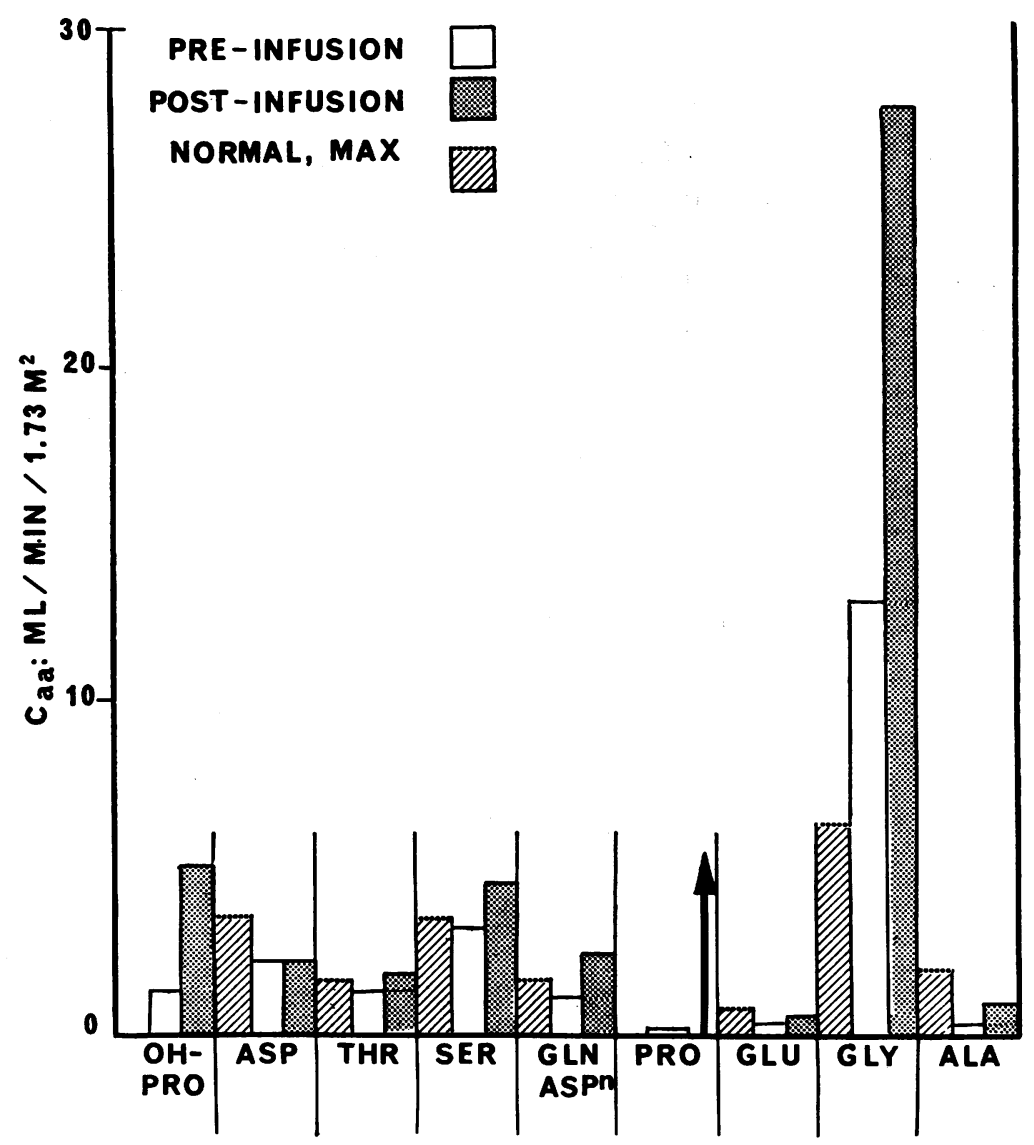

Fig. 3. Endogenous amino acid Renal clearances in a patient with FAMILIAL HYPERPROLINEMIA, BEFORE (CLEAR BARS) AND AFTER (45 TO 75 MINUTES) (STIPPLED BARS) IV INFUSION OF L-PROLINE. Upper range of values in normal subjects (Table II) is shown (hatched bars) for comparison. Preinfusion plasma proline concentration, $0.98 \mu$ mole per $\mathrm{ml}$; postinfusion (60-minute) value, $1.6 \mu$ moles per ml.

impaired glomerular and tubular function. $\mathrm{Hy}-$ droxyprolinuria and hyperglycinuria did not occur unless prolinuria was also present. In one patient who was studied in detail, endogenous amino acid renal clearance rates were determined before and in the third (45 to 75 minutes) period after rapid infusion of L-proline. The preinfusion plasma proline concentration was 0.98 $\mu$ mole per $\mathrm{ml}$, and there was abnormal iminoaciduria and glycinuria; urinary excretion of the other amino acids was normal. After infusion of proline, when the plasma proline was $1.6 \mu$ moles per $\mathrm{ml}$ (at 60 minutes), renal clearance rates for imino acids and glycine were further increased (Figure 3). Slight increases in threonine and serine clearances also occurred.
Therefore, the specific hyperaminoaciduria in familial hyperprolinemia is dependent on the hyperprolinemia, and the aminoaciduria has a mechanism similar to that in normal subjects receiving L-proline infusions.

The parents of the hyperprolinemic sibs were also investigated. The plasma proline levels and renal excretion of amino acids were normal in both subjects.

\section{Discussion}

Some reflection on the general properties of amino acid transport is of interest in discussing the foregoing experiments. Cellular transport of amino acids occurs by a facilitated or active process and not by passive diffusion (26). The 
transport process is rate-limited and exhibits specificity with reference to substrate (26). As would be expected, competitive inhibition for transport can occur between molecules of similar steric conformation. The properties of glycine transport in particular have been studied intensively (27-29), and in vitro observations on the high competitive affinity of imino acids for the glycine transport mechanism (27) have long preceded the present in vivo observations. The above-mentioned studies and many others like them have, in general, been performed on ascites cells or intestinal sac preparations. However, recent in vitro work $(30,31)$ has shown that the general principles apply equally well to amino acid transport in kidney slices. It should not be difficult then to find reasonable interpretation of the present in vivo investigation of renal tubular function in terms of the principles of amino acid transport.

Our initial observations in familial hyperprolinemia provided the stimulus for this investigation. These observations were that a specific hyperaminoaciduria accompanied hyperprolinemia, and that this aminoaciduria occurred only when the plasma proline concentration exceeded a certain limit. An explanation for these phenomena is now possible.

The amount of the filtered proline load determines the presence or absence of the aminoaciduria. Infusion studies in normal subjects showed that specific hyperaminoaciduria, indistinguishable from that of familial hyperprolinemia, accompanied an increase in the filtered proline load.

The mechanism of occurrence of this aminoaciduria had two distinct components. The prolinuria was dependent on the plasma proline level (i.e., filtered proline load); the threshold for prolinuria was the same in normal subjects as in familial hyperprolinemic subjects. Prolinuria in these subjects was therefore of prerenal origin (terminology of Dent and Walshe) (32). On the other hand, the hydroxyprolinuria and hyperglycinuria, which appeared when the filtered proline load was increased sufficiently, were the results of impairment of the tubular absorption; in other words, this component of the specific hyperaminoaciduria was of renal origin. This is apparently the first example of combined renal and prerenal mechanisms accounting for a specific hyperaminoaciduria in man.

The next point of interest concerns the significance of a threshold for prolinuria. The threshold itself was clearly detectable, unlike that for many other amino acids (reviewed by Smith) (33). Above threshold values, prolinuria rapidly increased, and eventually it became directly proportional to the filtered proline load. This observation suggests that the proline transport mechanism has a high affinity and a low saturation limit for its substrate; this characteristic has since been documented in vitro (31). Under specific investigation a maximal rate for tubular absorption of L-proline was found. This indicates that the tubular mechanism for transport of proline is saturable, as would be expected. The range of the TmPro, on the basis of the limited number of experiments, is fairly wide. The Tm was approached with some rounding off (Figure 2); this is also seen with many other substrates. The customary explanation is that such splay is indicative of heterogeneity in the nephron response to perfusion by the filtered substrate load (34). On the other hand, this rounding off of response is also a typical occurrence in the progression from first order to zero order kinetics, which is what prevails when saturation of a rate-limited transport reaction occurs.

The TmPro is, to our knowledge, the first amino acid $\mathrm{Tm}$ that has been demonstrated in man. Tm values for other amino acids in the dog have been observed (reviewed by Smith) (33).

The final point of interest arising from the investigations in familial hyperprolinemic and normal subjects concerns the specificity of the inhibitory effect that L-proline had on tubular absorption of other amino acids. This was limited to hydroxyproline and glycine; other manifestations of inhibition of transport were insignificant by comparison (e. g., of serine and threonine). Similar selectivity was noted when hydroxyproline was infused and thereby used as the inhibitor.

Steady-state infusion experiments with L-proline demonstrated that the inhibitory effect emerged only when the inhibitor was approaching the saturation level for its own transport. Thereafter, inhibition of glycine and hydroxyproline transport increased with increased filtered load of the inhibitor (L-proline). This suggests that pro- 
line has higher affinity for transport than glycine or hydroxyproline; when sufficient L-proline is bound to its transport mechanism, excess amounts become a competitive inhibitor against the latter two compounds. This particular data should indicate that proline, hydroxyproline, and glycine are not transported by a single transport mechanism. Initially, however, we postulated that the two imino acids and glycine shared a "common" transport mechanism for which the relative affinities of the three substrates were proline $>$ hydroxyproline $>$ glycine $(1,13)$. Inhibition of transport by a single member, limited to the group in its effect, should then be an indication of competition for the "common" system.

We found observations, reported elsewhere, in accord with the hypothesis that imino acids and glycine have properties of cellular transport in common. 1) In Hartnup disease $(11,12,35)$ impairment in renal and intestinal transport of most of the neutral amino acids is the result of a specific genetic mutation. However, transport of imino acids and glycine does not appear to be abnormal by comparison in this phenotype.

2) During the first few months of life, the human infant has elevated urinary excretion of imino acids and glycine, although the excretion of other amino acids is comparable to that observed in later life. This suggests a single mechanism responsible for the reabsorption of these three amino acids that is slow to mature in the infant kidney.

3) Joseph, Ribierre, Job, and Girault (36) described a convulsive disorder in infancy with a specific renal hyperaminoaciduria comprising proline, hydroxyproline, and glycine. Jonxis (37) has also studied a male infant with a history of neonatal convulsions, failure to thrive, and excessive urinary excretion of proline, hydroxyproline, and glycine persisting after early infancy. In both studies the plasma amino acid concentrations of the patients were normal. The aminoaciduria in these infants may therefore reveal impairment of a selective tubular reabsorptive mechanism for imino acids and glycine.

There are also, however, observations casting doubt upon the existence of a discrete transport mechanism "common" to imino acids and glycine: 1) In our experiments glycine infusion produced minimal prolinuria and no hydroxyprolinuria.
We tried to explain this by assuming very low affinity of glycine for the "common" system. The naturally occurring counterpart of this experiment is idiopathic hyperglycinemia $(38,39)$, in which there is a marked increase in renal filtration of glycine but no abnormal iminoaciduria.

2) Valine infusion increased glycine clearance but had no effect on imino acid clearances. If the imino acids and glycine were transported by a single common system, valine should inhibit all three substrates.

3) One subject in our study (the boy with hypophosphatemic rickets and hyperglycinuria) has impaired tubular reabsorption of glycine as part of his renal tubular syndrome, yet imino acids are not comparably affected. Furthermore, in familial (renal) glycinuria described by de Vries and associates (10), iminoaciduria was not observed.

4) In our experiments there were increases in clearance rates produced by the infusion of imino acids and glycine affecting amino acids beyond the limits of the hypothetical "common" transport system. Whereas glycine infusion had little effect on imino acid excretion, it increased clearance rates of serine and threonine, in addition to raising the plasma concentration of serine; this phenomenon has also been noted by others (40). Infusion of either imino acid increased clearance rates of serine, threonine, $\alpha$-amino-n-butyric acid, and the aromatic amino acids in later postinfusion periods. Clearance rates of serine and threonine were also slightly increased in familial hyperprolinemia (Figure 3).

Our initial concept (1) of a single (i.e., " common") transport mechanism specific for proline, hydroxyproline, and glycine does not fit all of the observations discussed. The difficulty appears to be in the belief that a "common" implies a "single" transport mechanism. Ahmed and Scholefield, who have recently investigated the criteria for proving that different molecules are transported at a single site (41), concluded that such a phenomenon occurs only under exceptional circumstances. Recently the transport of imino acids and glycine has been studied in vitro in mammalian tissue (31); apparently these three compounds are not transported by a single mechanism. Very interesting and important considerations are therefore raised concerning the structural and functional relations that must exist when separate 
transport mechanisms behave under certain genetic, developmental, and induced conditions as though they have something in common.

\section{Summary}

Renal tubular transport of imino acids and glycine was investigated in healthy human subjects and in patients with familial hyperprolinemia. In the latter condition there is a specific hyperaminoaciduria comprising proline, hydroxyproline, and glycine. This appears when the plasma proline concentration exceeds approximately $1.0 \mu$ mole per $\mathrm{ml}$ (normal maximum, $0.3 \mu$ mole per $\mathrm{ml}$ ). Identical hyperaminoaciduria was produced in normal subjects by iv infusion of L-proline.

Prolinuria occurs in healthy and familial hyperprolinemic subjects by a prerenal mechanism. Hydroxyprolinuria and hyperglycinuria occur by a renal mechanism, their tubular absorption being inhibited by high concentrations of L-proline. A combined mechanism for abnormal aminoaciduria has not previously been described.

Tubular transport of L-proline in man is a saturable process, and a TmPro is demonstrable. L-Proline becomes an inhibitor for glycine and hydroxyproline transport when it approaches the saturation limit for its own transport; the inhibition is presumably competitive. The transport of imino acids and glycine has interrelations not shared with other amino acids.

\section{Acknowledgments}

We are grateful to Miss Eluned Davies, Miss Ellen Matscheizik, Mr. Onslow Wilson, and Dr. F. X. Fellers for assistance with technical procedures. We are also indebted to Drs. Arnold G. Burgen, Francis Chinard, Charles E. Dent, Louis K. Diamond, Park S. Gerald, and Peter G. Scholefield for their interest and helpful discussions.

\section{References}

1. Scriver, C. R., I. A. Schafer, and M. L. Efron. Evidence for a renal tubular amino acid transport system common to glycine, L-proline and hydroxy-L-proline (abstract). J. clin. Invest. 1961, 40, 1080.

2. Cusworth, D. C., and C. E. Dent. Renal clearances of amino acids in normal adults and in patients with aminoaciduria. Biochem. J. 1960, 74, 550.

3. Webber, W. A. Interactions of neutral and acidic amino acids in renal tubular transport. Amer. J. Physiol. 1962, 202, 577.
4. Brown, J. L., A. M. Samiy, and R. F. Pitts. Localization of amino-nitrogen reabsorption in the nephron of the dog. Amer. J. Physiol. 1961, 200, 370.

5. Christensen, H. N., and J. C. Jones. Amino acid transport models, renal resorption and resistance to metabolic attack. J. biol. Chem. 1962, 237, 1203.

6. Webber, W. A., J. L. Brown, and R. F. Pitts. Interactions of amino acids in renal tubular transport. Amer. J. Physiol. 1961, 200, 380.

7. Frimpter, G. W., M. Horwith, E. Furth, R. E. Fellows, and D. D. Thompson. Inulin and endogenous amino acid renal clearances in cystinuria; evidence for tubular secretion. J. clin. Invest. 1962, 41, 281.

8. Robson, E. B., and G. A. Rose. The effect of intravenous lysine on the renal clearances of cystine, arginine and ornithine in normal subjects, in patients with cystinuria and Fanconi syndrome and in their relatives. Clin. Sci. 1957, 16, 75.

9. Arrow, V. K., and R. G. Westall. Amino acid clearances in cystinuria. J. Physiol. 1958, 142, 141.

10. De Vries, A., S. Kochwa, J. Lazebnik, M. Frank, and M. Djaldetti. Glycinuria, a hereditary disorder associated with nephrolithiasis. Amer. J. Med. 1957, 23, 408.

11. Baron, D. N., C. E. Dent, H. Harris, E. W. Hart, and J. B. Jepson. Hereditary pellagra-like skin rash with temporary cerebellar ataxia, constant renal amino-aciduria, and other bizarre biochemical features. Lancet 1956, 2, 421.

12. Milne, M. D., M. A. Crawford, C. B. Girão, and L. W. Loughridge. The metabolic disorder in Hartnup Disease. Quart. J. Med. 1960, 29, 407.

13. Scriver, C. R., I. A. Schafer, and M. L. Efron. New renal tubular amino-acid transport system and a new hereditary disorder of amino-acid metabolism. Nature (Lond.) 1961, 192, 672.

14. Schafer, I. A., C. R. Scriver, and M. L. Efron. Familial hyperprolinemia, cerebral dysfunction and renal anomalies occurring in a family with hereditary nephropathy and deafness. New Engl. J. Med. 1962, 267, 51.

15. Moore, S., and W. H. Stein. Procedures for the chromatographic determination of amino acids on four per cent cross-linked sulfonated polystyrene resins. J. biol. Chem. 1954, 211, 893.

16. Dent, C. E. Paper chromatography in medicine in Recent Advances in Clinical Pathology, 2nd ed., S. C. Dyke, Ed. Philadelphia, Blakiston, 1951, p. 238.

17. Efron, M. L. Two-way separation of amino acids and other Ninhydrin-reacting substances by highvoltage electrophoresis followed by paper chromatography. Biochem. J. 1959, 72, 691.

18. Kolor, M. G., and H. R. Roberts. A new reagent for the detection of hydroxyproline on paper chromatograms. Arch. Biochem. 1957, 70, 620. 
19. Spackman, D. H., W. H. Stein, and S. Moore. Automatic recording apparatus for use in the chromatography of amino acids. Analyt. Chem. $1958,30,1190$.

20. Moore, S., D. H. Spackman, and W. H. Stein. Chromatography of amino acids on sulfonated polystyrene resins. Analyt. Chem. 1958, 30, 1185.

21. Messer, $M$. Interference by amino acids and peptides with the photometric estimation of proline. Analyt. Biochem. 1961, 2, 353.

22. Schreiner, G. E. Determination of inulin by means of Resorcinol. Proc. Soc. exp. Biol. (N. Y.) 1950, 74, 117.

23. Miller, Z., and B. F. Miller. Specific determination of serum creatinine. Proc. Soc. exp. Biol. (N. Y.) 1951, 78, 471.

24. Kupiecki, F. P., and M. J. Coon. The enzymatic synthesis of $\beta$-aminoisobutyrate. A product of valine metabolism, and of $\beta$-alanine, a product of $\beta$-hydroxypropionate metabolism. J. biol. Chem. 1957, 229, 743.

25. Smith, H. W. The Kidney: Structure and Function in Health and Disease. New York, Oxford, 1958, p. 95.

26. Finch, L. R., and F. J. R. Hird. The uptake of amino acids by isolated segments of rat intestine. II. A survey of affinity for uptake from rates of uptake and competition for uptake. Biochim. biophys. Acta (Amst.) 1960, 43, 278.

27. Paine, C. M., and E. Heinz. The structural specificity of the glycine transport system of Ehrlich carcinoma cells. J. biol. Chem. 1960, 235, 1080.

28. Hagihira, H., T. H. Wilson, and E. C. C. Lin. Intestinal transport of certain $\mathrm{N}$-substituted amino acids. Amer. J. Physiol. 1962, 203, 637.

29. Spencer, R. P., J. Weinstein, A. Sussman, T. M. Bow, and M. A. Markulis. Effect of structural analogues on intestinal accumulation of glycine. Amer. J. Physiol. 1962, 203, 634.

30. Rosenberg, L. E., S. J. Downing, and S. Segal. Competitive inhibition of dibasic amino acid trans- port in rat kidney. J. biol. Chem. 1962, 237, 2265.

31. Scriver, C. R., and O. H. Wilson. Cellular transport of imino acids and glycine (abstract). J. Pediat. 1963, 63, 730.

32. Dent, C. E., and J. M. Walshe. Amino-acid metabolism. Brit. med. Bull. 1954, 10, 247.

33. Smith, H. W. The Kidney: Structure and Function in Health and Disease. New York, Oxford, 1958, pp. 104-109.

34. Bradley, S. E., and M. O. Wheeler. On the diversities of structure, perfusion and function of the nephron population. Amer. J. Med. 1958, 24, 692.

35. Scriver, C. R., and K. N. F. Shaw. Hartnup disease: an example of genetically determined defective cellular amino acid transport (abstract). Canad. med. Ass. J. 1962, 86, 232.

36. Joseph, R., M. Ribierre, J. C. Job, and M. Girault. Maladie familiale associante des convulsions à début très précoce une hyperalbuminorachie et une hyperaminoacidurie. Arch franç. Pédiat. 1958, 15,374 .

37. Jonxis, J. H. P. Personal communication, 1962.

38. Childs, B., W. L. Nyhan, M. Borden, L. Bard, and R. E. Cooke. Idiopathic hyperglycinemia and hyperglycinuria: a new disorder of amino acid metabolism. I. Pediatrics 1961, 27, no. 4, 522.

39. Nyhan, W. L., M. Borden, and B. Childs. Idiopathic hyperglycinemia : a new disorder of amino acid metabolism. II. The concentrations of other amino acids in the plasma and their modification by the administration of leucine. Pediatrics 1961, 27, 539.

40. Doolan, P. D., H. A. Harper, M. E. Hutchin, and E. L. Alpen. The renal tubular response to amino acid loading. J. clin. Invest. 1956, 35, 888.

41. Ahmed, K., and P. G. Scholefield. Biochemical studies on 1-aminocyclopentane carboxylic acid. Canad. J. Biochem. 1962, 40, 1101. 